Journal of Marital and Family Therapy

doi: 10.1111/j.1752-0606.2012.00322.x

January 2014, Vol. 40, No. 1, 92-105

\title{
COUPLE'S RELATIONSHIP WITH DIABETES: MEANS AND MEANINGS FOR MANAGEMENT SUCCESS
}

\author{
Ruth A. Houston-Barrett \\ Loma Linda University \\ Colwick M. Wilson \\ The University of Michigan
}

\begin{abstract}
Diabetes is a serious chronic illness that involves individuals, couples, and families in complex reciprocal interactions with the illness and other life dimensions. A major issue in diabetes management is how well the patient is able to follow the established medical regimen of selfcare. Situated within personal, physical, and social contexts, the meaning that a couple creates and the emotions they feel in their adaptation to living with diabetes and its demanding regimen can be viewed as the couple's relationship with diabetes.
\end{abstract}

The relationship between social support and chronic illness is well established and couple relationships seem to be particularly important to managing diabetes (Fisher, 2006). However, researchers have only begun to explore how specific types of support from a spouse are helpful or harmful to coping with diabetes management. Also, while a meaning-oriented, biopsychosocial approach to diabetes treatment has been widely promoted for researching chronic illness (Walsh, 1998; Wynne, Shields, \& Sirkin, 1992), few studies have adopted this framework to examine what makes couples successful in managing diabetes. This study views couples' processes of diabetes management in a biopsychosocial context.

\section{BACKGROUND}

\section{Diabetes Management}

Effective treatments for diabetes are vital, not only for 25.8 million people in the United States diagnosed with the illness (Centers for Disease Control and Prevention [CDC], 2011), but also for their family and friends. Diabetes doubles the risk of death; it was the seventh leading cause of death in the United States in 2007 (CDC). It has risks of serious complications, including blindness and amputation, and is associated with decreased quality of life. The CDC estimated the 2007 cost of diabetes in the United States at \$174 billion. Additionally, diabetes rates are climbing dramatically: in a national sample, the age-adjusted incidence of diabetes increased 90\% from 1995-1997 to 2005-2007 (Kirtland, Li, Geiss, \& Thompson, 2008). If current trends continue, 1 in 3 Americans will develop diabetes in their lifetime and, on average, will lose 10-15 years of life (CDC, 2009).

A major issue with diabetes is how well the patient follows the established medical selfcare regimen for diet, exercise, medication, and blood glucose testing (McCaul, Glasgow, \& Schafer, 1987). Research suggests that adherence to this regimen is low, and interventions to improve adherence have been largely unsuccessful (Peyrot et al., 2005). It seems that while people with diabetes believe it is a serious condition, many are able to reconcile that belief with less than full adherence to medical lifestyle advice (Murphy \& Kinmonth, 1995).

Ruth A. Houston-Barrett, PhD, School of Behavioral Health, Loma Linda University; Colwick M. Wilson, $\mathrm{PhD}$, The University of Michigan, School of Nursing.

The first author would like to acknowledge Professor Carmen Knudson-Martin, LMFT, Director of the Doctoral Program in Marital \& Family Therapy, Loma Linda University for her invaluable assistance with the early development of theory in this paper.

Address correspondence to Ruth A. Houston-Barrett, School of Behavioral Health, Loma Linda University, 11226 Campus Street, Loma Linda, CA 92350; E-mail: ruthhouston.barrett@gmail.com 
Diabetes is also challenging for its complex, interactive effects on health, relationships, and other psychological, contextual, and social factors, which often make blood sugars fluctuate in largely unpredictable ways. Accordingly, current research has expanded from a focus on physical management to include critical relational, emotional, and meaning-oriented aspects. Family and couple relationships in particular have received increased attention, with results indicating their substantial importance to diabetes management (Fisher, 2006).

\section{Close Relationships and Chronic Illness}

The establishment of the reciprocal relationship between social support and chronic illness (Kowal, Johnson, \& Lee, 2003) has added to the growing recognition that family dynamics impacts health outcomes (Campbell, 2003; Williams, Frankel, Campbell, \& Deci, 2000). Increasingly, research of how families and couples cope with stress is expanding individual-focused research orientations to include relational perspectives (Kayser, Watson, \& Andrade, 2007).

Studies of close relationships and illness have consistently found that illness management is associated with relationship satisfaction, conflict, criticalness, hostility, and congruence in beliefs and expectations concerning the disease (Fisher, 2006). For diabetes in particular, when family members support care regimens, more satisfaction with adaptation to diabetes and less interference in role function are reported (Trief, Grant, Elbert, \& Weinstock, 1998).

\section{Couples Relationships and Diabetes Management}

Studies have shown that couples' relationships, including their marital satisfaction, are important to diabetes management (Fisher, 2006). Specifically, feeling emotionally supported by the spouse significantly predicts regimen adherence and lifestyle satisfaction (Trief, Wade, Britton, \& Weinstock, 2002). Studies have also examined support in terms of the diabetic's perception of spouse's beliefs (Miller, Wikoff, Keen \& Norton, 1987) and expressed emotion (Wearden, Tarrier, \& Davies, 2000), finding them to be important predictors of regimen adherence and glycemic control.

Support can also be viewed as a couple's coping phenomenon: how couples adapt and cope together in living with illness. A qualitative study found that resilient couples had a mutual coping philosophy and an "in it together" attitude (Skerrett, 1998). It was beneficial when couples adopted a collaborative coping style (Berg et al., 2008). Also, illness management was related to how couples appraised and responded to the stress, coordinated their coping efforts, and found meaning in the experience (Kayser et al., 2007). Together, these studies reveal that collaboration, mutuality, and meaning-making are important to illness management.

\section{A Meaning-Oriented, Biopsychosocial Approach}

A significant challenge to contemporary treatment of diabetes is overcoming the many psychosocial and environmental barriers to optimal self-management (Gonder-Frederick, Cox, \& Ritterband, 2002). The biopsychosocial model is widely promoted to address the complex issues around physical illnesses (Kowal et al., 2003; McDaniel, Hepworth, \& Doherty, 1992; Snoek \& Skinner, 2002). Further, a meaning-oriented approach that views diabetes as a narrative in a biopsychosocial context is promising (Wynne et al., 1992). In this vein, medical providers are urged to incorporate narrative and postmodern perspectives into their work with diabetics and to collaborate with patients and families around personal belief systems and expectations (Delamater, 2006; Snoek \& Skinner, 2002).

Recent outcome research for diabetes management has shown the value of addressing meaning construction about illness experiences. Diabetes selfcare is affected by perceptions, attitudes, meanings, and beliefs (Snoek \& Skinner, 2002); image of the disease, meaning of the diagnosis, integration of the illness, and space for the illness (Hornsten, Sandstrom, \& Lundman, 2004); irrational health beliefs and personal goals and priorities (Talbot, Nouwen, Gingras, Bélanger, \& Audet, 1999); explanatory models (O’Connor, Desai, Solberg, Rush, \& Bishop, 2003); and stories of selfefficacy, autonomy, and competence (Williams, McGregor, Zeldman, Freedman, \& Deci, 2004).

Meaning-making processes and perceptions are personal within the context of one's culture and are influenced by factors such as race, gender, and age cohort. For instance, African 
Americans described having a greater sense of loss associated with diabetes than their white peers (Ford, Havstad, Brooks, \& Tilley, 2002); women patients with heart failure ascribed more positive meanings to their illness than men (Evangelista, Kagawa-Singer, \& Dracup, 2001); and the explanatory models of older diabetic patients seemed more beneficial than those of their younger cohorts (O’Connor et al., 2003).

One of the most beneficial of illness meanings seems to be that which is transforming: profoundly positive shifts in perspective about the illness and its consequences. That is, some participants with diabetes described how a transforming experience arose from interpreting a challenge in such a way as to "create a new relationship with the illness... mediating the impact of disease by altering one's cognitive and affective response to it" (Paterson, Thorne, Crawford, \& Tarko, 1999; pp. 799). Similarly, HIV-positive men who held a transforming view of HIV as a catalyst for personal growth coped more effectively than those who viewed HIV as punishment (Schwartzberg, 1993).

While it is clear that couples' meaning-making and supportive interactions are important to managing chronic illness, there is a great deal yet unknown about the specific dimensions that foster couples' success, especially in managing diabetes. This study used qualitative interviews with couples to access their experiences, focusing on the emotions and meanings that form couples' relationships with diabetes, and on couples' coping and supportive interactions in managing diabetes care requirements. This study generated theory regarding how these dyadic constructs interact with success in diabetes management.

\section{METHOD}

We used a qualitative approach to obtain new perspectives and in-depth understandings about how couples manage diabetes (Strauss \& Corbin, 1998). We used a grounded theory approach to develop explanatory theory about social processes and meaning-making that affect management success, in the context of couples' experiences of living with diabetes (Starks \& Trinidad, 2007). We employed Strauss and Corbin's (1998) coding techniques for grounded theory development to organize the raw data into abstract concepts and to find patterns and relationships among these concepts. This approach emphasizes that the meaning emerges from the data, rather than from preconceptions, negotiated as researchers engage with the text. The researchers' epistemology, politics, and ethical stance drive the process of "making sense of one's findings" (Denzin \& Lincoln, 2000, pp. 23). Validity is a function of how well researchers construct "an authentic and compelling narrative of what occurred in the study and the various stories of the participants" (Janesick, 2003 pp. 58).

Adopting a critical theoretical perspective, we give participants' intimate, practical knowledge of their own experiences the greater (truth) status in the interactive construction process (Gergen, 1999). As family therapists, we view diabetes as located within a relational, systemic context. From a social constructionist perspective, we highlight meaning as being of primary importance in understanding how persons live with diabetes. Meanings, attitudes, and beliefs significantly impact quality of life, relationships, and illness management (Hornsten et al., 2004). Thus, participants' perspectives and meanings were central to our research question and theory development, and we used direct quotations from the interviews as the data source (Hill \& Thomas, 2000).

The first author was a Marriage and Family Therapy (MFT) PhD candidate, and the second author was a professor and head of an MFT doctoral program, at the time this paper was written. Both researchers have strong interests and several years experience in diabetes research, particularly as framed by relational and systemic orientations. The first author brings a perspective as a narrative and collaborative language systems therapist through which she seeks meaning construction in the language of participants. She also has a deep, ongoing interest in psychosocial influences on chronic physical illness. The second author has an added perspective as an insider, having family members with diabetes.

\section{Sample}

This study was focused on the ways that couples with diabetes make sense of medical directives in the context of their culture, history, and relationships. We used theoretical 
sampling (Strauss \& Corbin, 1998) to ensure that the data showed sufficient, meaningful variation in the emerging categories to highlight patterns and relationships leading to theory development. We chose couples as our unit of analysis, hoping to learn about their emotions, meaning-making, and relational interactions in diabetes management. Couples in this study were married or living together for at least 2 years.

The sample includes couples from diverse ethnic and cultural backgrounds, a broad range of ages and educational levels, and multiple religious traditions. The 50 participants were composed of 25 heterosexual couples with 27 diabetics. All, but one were married, with relationship lengths ranging from 4 to 56 years (mean 29 years). Ages ranged from 24 to 79 years (mean; 54.9 women, 56.5 men). There were 18 Whites, 17 Hispanics, six Blacks, two Pacific Islanders, two Filipinos, two Armenians, two Iranian Armenians, and one Anglo-Native. Educational levels ranged from high school to graduate school. Fifteen individuals were retired, seven were employed in the medical field, six were housewives, and 22 held a variety of other occupations.

\section{Recruitment}

We invited participants to be part of a study on "Diabetes and Relationships" conducted by Loma Linda University, recruited through fliers, from patient groups at a Diabetes Treatment Center, and through "word-of-mouth." Criteria for inclusion were one or both spouses diagnosed with Type 2 diabetes and both willing to be interviewed together. We invited couples to share their stories about their experiences with diabetes in their lives and relationships. We followed procedures for protecting participants that were approved for this study by the Loma Linda Institutional Review Board. This included obtaining informed consent of participants with description about how confidentiality would be protected, as well as possible harm that might arise from discussing emotional issues in the interview. We provided referrals for lowcost counseling services, made it clear that the interview could be stopped or questions could be skipped at any time, and we paid close attention to accommodate needs or tensions that might arise when interviewing the spouses together.

\section{Interviews}

Data collection used an interview guide of broad questions structured so that data across participants would be comparable (Hill \& Thomas, 2000), while allowing participants to discuss what was most important to them. Our questions included enquiries about their relationship; personal context; diabetes and its impact on self, others, and interactions with medical professionals; and history, such as family experiences with chronic disease. For example, we asked, "How does diabetes affect your relationship?", "What kinds of decisions have you had to make about your diabetes?", and "Who worries most about the diabetes?" Researchers probed to elicit richer responses. For example, following one couple's response to the general question, "How do you balance the needs of the relationship with individual needs?" the interviewer probed, "What does that mean to you, when he lets you do something for him?"

Interviews lasted between $1 \frac{1}{2}$ to $2 \mathrm{hr}$ and were audiotaped and transcribed. All personal identifiers were deleted, and names used are pseudonyms. Interviews were conducted by the author and other doctoral students enrolled in a qualitative research class.

\section{ANALYSIS}

Consistent with grounded theory (Strauss \& Corbin, 1998), the study began with no predetermined analytic categories. Interviews were examined with a broad interest in what participants described about living with and managing diabetes, to capture as much information about what makes it easier or more difficult to manage diabetes. We used open coding, reading transcripts line-by-line and identifying and labeling concepts such as, "she eats what he eats," "fear is a motivation," and "husband listens to wife's management directives." Similar items were assigned the same label, while different items received a new label. In this constant comparison process, categories arose from the data, rather than from researchers' a priori expectations.

Next, we used axial coding (Strauss \& Corbin, 1998) to group labels hierarchically, according to dimensions/range of broader/more abstract categories. In this way, we grouped labels 
about couples' supportive interactions around diabetes management into three categories: "partnering/doing it together," "pushing/reminding," and "uninvolved," which in turn evolved into a single, broader category called the couple's comanagement style. Similarly, we grouped meanings and emotions about living with diabetes into categories of positively transforming, matter-of-factly accepting, and adversely rejecting, and together these became the core category: the couple's relationship with diabetes.

In the final level of analysis, we employed selective coding to develop grounded theory about how these categories interconnect and influence each other and how they make sense in the context of the couples' narratives about living with diabetes. Our core question was: what makes couples more or less successful in achieving diabetes management?

To create grounded theory about this question, it was essential to have a consistent means of evaluating degrees of management success in this study. To compare this dimension among the couples, we first had to be clear about what we meant by "more or less successful in diabetes management." We were not attempting to increase depth of understanding about this concept, which is well-defined in the literature to be comprised of adherence to medical recommendations in four areas: exercise, diet, medications, and testing (McCaul et al., 1987). Rather, we were focused on finding out what in a couple's experience would affect this relatively concrete phenomenon.

To be as direct, consistent, clear, and meaningful as possible, we developed a semiquantitative scoring method, as described below. Semiquantitative coding has been used rather widely in the general scientific literature, but we did not uncover any instances of using this technique in combination with the Strauss \& Corbin methodology. However, we feel that the application of semiquantitative coding in our analysis provides parsimony and clarity, without truncating relevant meaning.

We identified and coded management success in the four aspects of regimen adherence. We then employed semiquantitative coding (Johnson, Dunlap, \& Benoit, 2010) by assigning two points for "consistent," one point for "inconsistent," and zero points for "none/rarely" in each of the four selfcare areas, and summing these four subscores to create a numerical representation of diabetes management success. Thus, the highest possible score of 8 points was given to couples who described being consistent in all four dimensions of selfcare. The lowest score in our sample was 2, such as for Estella, a 43-year-old Mexican-American mother of three who hated and feared glucose testing: "I don't want to know . . . I don't check myself my sugars..."

\section{Core Category and Grounded Theory Development}

Once we implemented our scheme to consistently and clearly categorize couples in terms of their diabetes management success, relationships emerged with and among other important categories describing the couples: their relationship with diabetes, comanagement style, attributions of responsibility, interactions with medical professionals, influences of friends and family, and talk about diabetes. As theory developed, it became evident that the core category related to management success through which all others were linked was the couple's relationship with diabetes: their cohesive, emotion-laden narrative about living with diabetes. We asked questions that deepened and refined our understandings of how the other categories linked to our central category, especially in terms of what made diabetes management more successful or difficult. In this way, we developed an explanation of how our core category interacted with other categories. The following results present our grounded theory of how the couple's relationship with diabetes frames and interacts with other constructs.

\section{RESULTS}

Three types of couples' relationships with diabetes emerged: transforming, accepting, and rejecting. Our grounded theory explains how the type of relationship with diabetes impacts diabetes management success as well as other aspects of living with the illness. First, we describe our grounded theory. Then, we provide detailed illustrations and evidence for the theory from the study data. 


\section{Grounded Theory: the Couple's Relationship with Diabetes}

The couple's relationship with diabetes is a cohesive set of emotions and meanings that the couple creates about diabetes and its management. It can be positive (transforming), neutral (accepting), or negative (rejecting). It is dynamic and may vary over time in response to couples' ongoing experiences of living with the disease, and it may buffer or exacerbate reactions to those experiences. It manifests cognitively (how they think about, understand, and plan for diabetes), physically (how they manage selfcare), emotionally (how they feel about living with diabetes), and socially (how they interact with each other around diabetes, as well as with other family members, friends, and medical professionals). It may be cohesive within the couple, a shared construction of meaning, or it may be disparate, even conflictual.

Of particular significance, the couples' relationships with diabetes were very much associated with their diabetes management success. Those with the most positive, transforming relationships achieved excellent management (scores 8 of 8). Those with accepting relationships had fair-to-excellent management (scores 5-8). Those with rejecting relationships with diabetes were experiencing grave difficulties in managing diabetes (scores 2-3).

Also important to the couple's life with diabetes is their comanagement style: partnering ("let's do it together"), pushing ("you should do it"), or uninvolved ("it's your problem, not mine"). Partnering couples did most of the management tasks together, especially for diet and exercise. In pushing couples, the non diabetic spouse reminded and encouraged the diabetic spouse to follow the diabetes regimen, but they rarely carried out the tasks together. In uninvolved couples, the diabetic spouse managed primarily alone, and the nondiabetic spouse avoided the business of diabetes care, at the insistence of either or both spouses. Management success was generally highest for the partnering couples, and lowest for the uninvolved couples.

Participant couples spoke about how their relationship with the illness had evolved. In turn, their relationship with diabetes framed and made sense of their experiences of living with diabetes and its demanding selfcare regimen, shaping how they managed the illness as a couple.

We propose the grounded theory (see Figure 1) that the couple's relationship with diabetes frames, influences, and manifests in their comanagement style, attributions of responsibility, interactions with medical professionals, interactions with friends and family, and talk about management achievement and difficulties. Taken together, these have a great deal of influence on the couple's success in diabetes management. The couple's relationship with diabetes shapes their emotions and actions regarding diabetes management, as well as framing

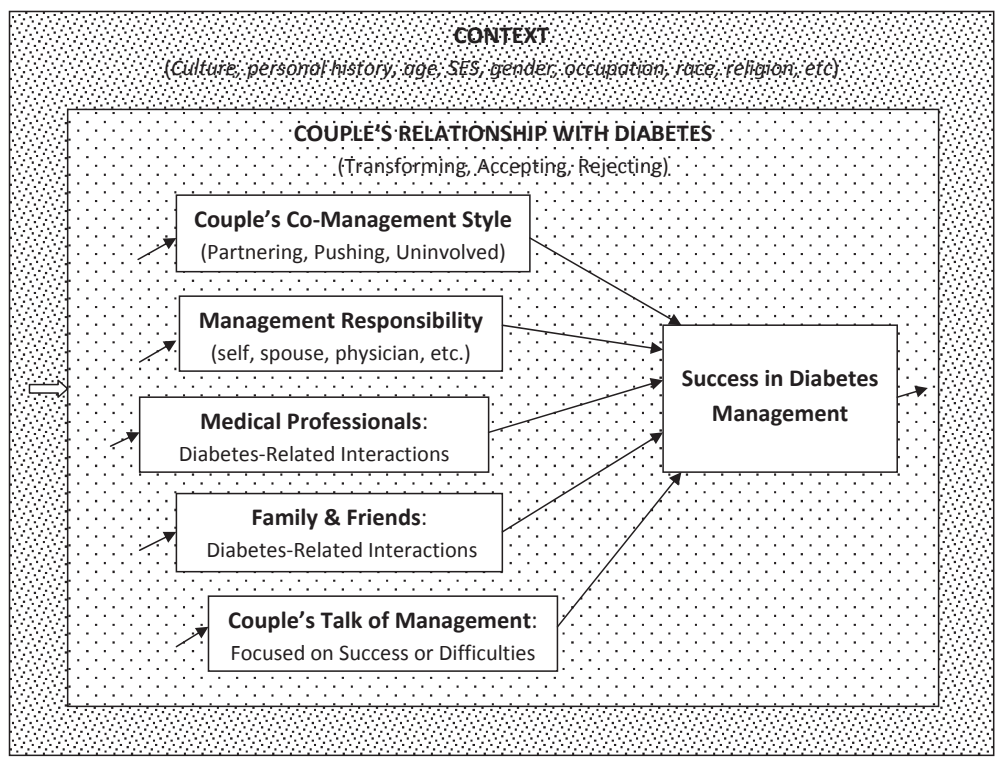

Figure 1. Grounded Theory Model of the Couple's Relationship with Diabetes. 
their perceptions of diabetes experiences, which, in turn, influence the relationship with diabetes to evolve.

In the following discussion, we provide evidence for our grounded theory with rich descriptions of the participant couples, grouped according to their type of relationship with diabetes: transforming, accepting, or rejecting. These illustrations show how the relationship with diabetes appears to frame and shape their success in diabetes management, and how it is influenced by and manifested in other aspects of living with the illness: comanagement, responsibility, social interactions, and talk about management success or difficulties.

\section{A Transforming Relationship with Diabetes}

About one-fourth of participant couples had transforming relationship with diabetes. These couples told narratives of diabetes bringing profound benefits to their lives, such as being a crucial wake-up call for their health or an epiphany of being loved. Couples in this group were highly successful partners in diabetes management, with scores of 8 of a possible 8 .

One couple with a transforming relationship with diabetes was Les, a publishing company president, and Kris, a "social and domestic engineer," both Caucasian and with four children. Les depicted diabetes as a life-saving blessing: "I've always looked at it, once I got over the initial shock, as a blessing, 'cause I was so heavy back then, I probably would have been dead by now... It's caused me to take care of myself." Another transforming couple, Franklin and LaNae, medical professionals, African-American, in their 50s, married 26 years, felt similarly: "It was by Design. Because it really helped in the way it brought us closer together."

Comanagement style in transforming couples: partners. Three comanagement styles were identified in this study: partnering, pushing, and uninvolved. Couples with transforming relationships with diabetes had partnering comanagement styles, working actively together to manage diabetes. One such was Mango and June, married 33 years ago in the Philippines, where Mango was a cop for 16 years. They immigrated to the United States shortly after being married and had two sons. Mango liked that his wife June took a partnering role, managing his diet, cooking for him, and "She comes 30 min with me just walking, just to get a little bit of exercise." They were also partners in spirituality, "Number one is that we pray together to have me ... in good health."

Responsibility in transforming couples. Each of the transforming couples, while partners in management, attributed ultimate responsibility to the diabetic spouse. Kris and Les illustrated this balance when she admonished, "I refuse to ever be a nag," and he said that this "caused me to take responsibility, myself. . You have to come up with your own unique system for what works for you." Another transforming, partnering couple was John and Janice, an electrician and a fitness instructor, Caucasian, in their 40s, married 19 years. Janice expressed similar thoughts about her diabetes, "The best treatment is taking responsibility for your actions. . . find what works for you."

Interactions with medical professionals in transforming couples. About half of the transforming couples had positive general attributions about medical professionals, and all felt positively about their own. For example, Mango and June liked how Mango's physicians and nurses "treat me as. . an older brother. . They give me lots of . . peace of mind." It seems that a transforming relationship with diabetes may invite positive interactions with medical professionals, and helpful interactions with medical professionals are likely to be conducive to more successful relationships with diabetes.

Friends and family of transforming couples. A transforming relationship with diabetes seems to helpfully influence diabetes-related interactions with friends and family, and vice versa. That is, Franklin valued his friends' advice: "Some people would have spent hundreds of dollars to get the information that was readily and freely and eagerly given to me." He also described how his son affected his relationship with diabetes, "It was really a wake-up call. He told me, 'Your toes fall off.' And, you know, that was really an example for me to really take this thing really, really seriously." Janice also was affected by her son's reaction to her diagnosis, "It just struck, Oh my God, my son loves me!" 
Talk about success or difficulties in transforming couples. Couples who had transforming relationships with diabetes often shared stories about how they were able to manage diabetes successfully and did not dwell on what made it difficult. For example, John and Janice spoke of their strategy, (Janice) "Planning: just always make sure that I have what I need," and (John) ". . . if you think ahead and calculate and plan."

\section{An Accepting Relationship with Diabetes}

Just under a third of the couples had an accepting relationship with diabetes. These couples spoke about the disease in a matter-of-fact language, neither greatly positive nor greatly negative. For them, the diabetes was not so much welcomed as accommodated. They spoke consistently of the need to accept that diabetes was not going away and that living with it simply required following the prescribed regimen. These couples experienced excellent-to-fair diabetes management, with scores ranging from 5 to 8 of a possible 8 .

Part of the accepting relationship seemed to be a matter-of-fact attitude that diabetes requires certain things daily. Maria and Carlos, a Mexican-American couple married for 43 years, exemplified this when Maria said, "I take my blood monitoring. I take my medication. . . . It's just like brushing your teeth. . . . No major thing.” Carlos agreed , “. . . she was in medical field, so she knows the avenues to take for her system, so it really didn't affect me at all."

Two couples fluctuated between accepting and rejecting relationships with diabetes, leaning more toward accepting; we called them partially accepting. They had poor-to-fair diabetes management scores ( 3 and 4 ). One couple, Reuben and Sui, married almost 50 years with three children, were both diabetic. They emigrated from Cuba 21 years ago, where he was a physician, and she a nurse. Reuben expressed rejecting, then accepting, in the same breath: "My goal is of getting rid of my diabetes; living with it and living a healthy life is my main goal."

Comanagement style in accepting couples. Couples with accepting or partially accepting relationships with diabetes were almost evenly split between partnering and pushing comanagement styles. A pushing style is one in which the nondiabetic spouse reminds, encourages, nags, or pushes the diabetic spouse to follow the prescribed regimen. Only two of the 13 accepting couples had an uninvolved style, in which they ignored, rejected, or avoided management tasks and needs.

Pilar and Alfonso had a partially accepting relationship with diabetes and a pushing comanagement style. Married for 45 years, in their 70s, with four grown children and four grandchildren, they had emigrated from Peru 3 years ago. Pilar expressed concern for her husband's health through pushing: "I worry a lot when I see him eating a lot of that cake, and I tell him, 'Don't eat too much." Alfonso liked the concern, "They worry because they love."

Responsibility in accepting couples. In accepting couples, most diabetic spouses took responsibility for their own illness management, with more success than the two who viewed it as their spouse's responsibility. They also had better management than the partially accepting couples, in which responsibility and blame were attributed to their physicians, wives, or culture.

Samuel and Margaret, Caucasian, in their 60s, with two adult sons, had an accepting relationship and pushing style, and preferred her to be responsible for his diabetes management. His management was fair: consistent in testing and medications and inconsistent in diet and exercise, and he was experiencing loss of use and numbness in his hands, bleeding, and sexual dysfunction. Asked who was the most worried about his diabetes, Samuel replied, "Probably she is. . .She is on top of it. . She says, "Are you sure you have to eat that?"' Margaret agreed, "If I don't watch him, he gets off." Unlike the dynamic in rejecting couples, Samuel accepted his wife's influence: "We discuss it. And most of the time, she is right."

Interactions with medical professionals in accepting couples. All of the couples who had accepting or partially accepting relationships with diabetes liked their own doctor, and most had positive opinions of medical professionals in general, such as Giovani, "I think that most doctors are good." Others, such as Reuben and Sui who had a partially accepting relationship, liked their current physician, but not others, as Sui complained they "don't take the time to 
talk to you." It seemed consistent with their accepting relationship that they had persisted until they found a doctor they liked and medical care that met their needs.

Friends and family of accepting couples. Most accepting couples said that family members also seemed to accept and accommodate the illness needs. Graciela described her family, "When I was diagnosed with diabetes, everyone bent over backwards to accommodate what things were appropriate and advised by the doctor. . There's always diet soda."

Talk about success or difficulties in accepting couples. Most of the accepting couples spoke more about how they succeeded with diabetes management than about why they could not. Giovanni explained his strategy, "I find that I have to plan things out a little bit more than I used to." Some difficulties were also described; for example, Sui said, "If he loses the weight then he would lose the diabetes . . but right now that's impossible because of his foot. He ... can't do anything."

\section{A Rejecting Relationship with Diabetes}

The three couples who had a rejecting relationship with diabetes spoke about the disease in very negative terms, often with fear or anger, refusing to accept the requirements, symptoms, or consequences of diabetes. Three other couples fluctuated between accepting and rejecting, leaning toward rejecting: we called them partially rejecting. Another couple's talk about diabetes indicated a relationship with diabetes that vacillated from transforming to rejecting.

The six couples who had rejecting or partially rejecting relationships with diabetes had the lowest scores for diabetes management success, ranging from 2 to 3 (out of 8). Further, two of the three couples with rejecting relationships had the very lowest scores in our sample (2). The couple that varied from transforming to rejecting had a management score of 6 .

Gordon, a teacher, and Kaila, a marriage and family therapist, are of Hawaiian heritage, in their 40s, together for 32 years, with four children. Gordon had been on flight status in the military until a physical revealed his diabetes 12 years ago. He did little to manage diabetes: "I eat what I want. . .I don't test like I should." He expressed their rejecting relationship with diabetes, "It seems like I try to. . .put it off and forget about it and hopefully it will go away."

For Hector and Estelle, Mexican American in their 40s with three children, a salesman and a fulltime homemaker, their rejecting relationship with diabetes seemed to arise from fear. Estella expressed, "I don't listen nor read much about diabetes. . .I know I have it, but I don't want to know." Hector explained, "It. . .makes her nervous to know that she has to check herself. It is not because of the prick, it is looking at the result, and it affects her a lot." It was rejecting from the start, Estella described, "At the beginning I was in denial. . .I said, "No, that is not! . . And they gave me medication and I didn't take it. Because my denial was so much."

Ali and Uma's rejecting relationship with diabetes formed from loathing of the regimen and rage at the serious consequences. In this Iranian-Armenian couple, ages 70 and 67, Ali was diagnosed 31 years ago, near the time he married Uma, and they emigrated from Tehran 6 years later. He expressed anger that "all comes from the sickness. Affects my eyes, affects my nerves. Affects my heart, affects my kidneys, affects my legs. . That is what makes me upset and angry." He rejected it from the start, "There was supposed to be a diet, but I was not paying attention. People warned me, but I . . thought that the worst wouldn't happen to me." Uma could not influence her husband: "Because he doesn't listen."

Comanagement style in rejecting couples. Couples with rejecting relationships had uninvolved comanagement styles. Those with partially rejecting relationships varied in style, from uninvolved, to pushing, to partnering. The uninvolved spouses expressed preferring to remain uninvolved, or they talked about helping, but in a vague and ineffectual way, or they accepted that their spouse would not let them help.

Juliana and Cal, in their 20s, married 6 years with two small children, had a rejecting relationship with diabetes and an uninvolved comanagement style. Juliana said her diet irritated Cal: "When I go grocery shopping, I put the fresh fruits and vegetables in the basket, and he says 'You are just buying that for you, nobody else eats that." Meal preparation was difficult: "It's hard to make a meal that I can have, that he will eat. That's the hardest thing . . part of the reason I don't have control." About her insulin shots, Cal said, "When she does it next to me, the smell. . the nastiest smell." When Juliana was irritable from high blood sugar, Cal 
said, "I usually just snap back at her." When asked whether he did anything to help her with diabetes, he replied, "I don't think so." Juliana wished him to be more involved, "What would help is having both the spouse and the patient at the educational teachings."

Responsibility in rejecting couples. In the six rejecting or partially rejecting couples, three felt the diabetic was responsible for selfcare, and three attributed responsibility or blame to the spouse, physician, or culture. Jaime, a construction supervisor, and Sarah, a county employee, in their 40s with two children, were partially rejecting and had poor management (score 3 of 8 ). They felt that it was Sarah's responsibility to manage his diabetes: (Jaime) "She is always on me about eating healthy," and (Sarah) "I'm like an angel." They also blamed doctors: "They didn't give him good care at Loma Linda, they didn't, they would say, 'Here are your pills,' and 'See you in a month!"'.

Interactions with medical professionals in rejecting couples. Only half of the couples who had rejecting or partially rejecting relationships with diabetes held generally positive opinions of medical professionals. Even for couples in this group who liked their physicians and agreed with medical advice, the rejecting relationship with diabetes seemed to interfere with the actual implementation of that advice. For example, Ali had a largely negative view of physicians: "They only think about the money," but liked his own, "Whatever they say ... I believe, I do it," yet his diabetes management was very poor, scoring 2 of 8 (inconsistent in medications and testing, rarely following diet and exercise advice).

Friends and family of rejecting couples. A rejecting relationship with diabetes seemed to negatively influence interactions with friends and family, and vice versa. Uma described how Ali's rejection of diabetes created problems between them, "There is always a problem. . All the time he curses, and says, 'Why do You give this sickness to me?"' For Faith, a dental assistant, and Scott, an operating engineer, diabetes-related emotions and interactions between them impacted their relationship with diabetes, which varied from transforming to rejecting. Faith worried that her diabetes might affect Scott, "that he wasn't gonna love me as much, you know (starts to cry). Because it's a challenge, it is." This worry led to rejecting diabetes selfcare (score 6), "I get into a depression, and I feel that I'm not. . .it's a lot of work, and I just don't feel I have the strength to do it."

Talk about success or difficulties in rejecting couples. Most of the talk about diabetes management in the partially or fully rejecting couples was about difficulties. For example, the diabetes diet conflicted with Gordon and Kaila's Hawaiian culture (Gordon): "The doctors already told me that I should cut down on eating rice, and I would not do that . . In our culture . . . rice is the main staple." Kaila agreed, "If I were to try to serve him brown rice, oh, my gosh!" Also, Juliana blamed American culture for the use of sugar, "They get it [sugar] from school, or kid's parties, I mean, it's out there. We even do it."

\section{Relationships with Diabetes are Dynamic}

The couples' relationships with diabetes were dynamic and evolving. Positive experiences with diabetes management generally led to more positive relationships with the illness. Negative experiences often resulted in more negative relationships, when felt as fear, avoidance, or resignation, but they sometimes influenced toward more positive relationships, when viewed as a wake-up call or epiphany.

Positive relationships seemed to buffer negative experiences, while negative relationships seemed to exacerbate them. For example, when Estella was unable to get control of her blood sugar, she felt fear and loathing that increased her rejection of diabetes. In contrast, John and Janice's philosophy helped them to overcome setbacks, reinforcing their transforming relationship: "You identify and take care of what is in your control and let go of what is not."

At times, a transforming relationship arose from a vivid realization or shock. Franklin spoke about the profound effect that his diagnosis had on the couple: "So when I went to the doctor's office, basically from there I came home and told LaNae what was happening, and we just cried. That was the first day of what I consider a new beginning." For Jorge and Juanita, a Mexican American couple, the shock of his losing vision in one eye was a wake-up call that changed their rejecting relationship to accepting. At first, Jorge said, "I never listened. I was still drinking, smoking. . .I didn't take it seriously." After this, he said, "That when I started 
caring for myself." Juanita explained, "Sometimes is hard, it takes something dramatic had to happen."

\section{Personal context and Culture, Gender, Occupation, Religion}

Context is a final, important consideration in this study. Each couple is situated in a personal context that includes culture, race/ethnicity, gender, SES, occupation, and religion. Context makes up much of personal reality, including what seems possible and desirable. It greatly affects meaning-making processes, such as the development of the couple's relationship with diabetes. In this study, culture, gender, occupation, and religion were of particular note, and while our sample is too small to make generalizable conclusions, these phenomena do point to the idea that the intersection of identities and contexts is important to diabetes management.

Gender and occupation together may play a role in the meaning-making processes. Men and women were similarly distributed across degrees of success in diabetes management. However, in the intersection of gender and occupation, the two diabetic spouses who were female and whose occupation was "housewife" had very poor diabetes management. Similar results were not found for diabetic husbands of housewives. Gender may affect meaning-making processes: in two couples, the husband's rejecting relationship with diabetes was strongly dominant to the wife's. Also, all the couples in which one or both were medical professionals were successful in management (scoring 7 or 8), with transforming or accepting relationships with diabetes.

Many spoke of spirituality or faith helping them to cope better with the stress of diabetes. However, religious talk was equally present over all types of relationships with diabetes, and it was not associated with management success. Other studies demonstrate more nuanced differences in how religion may be helpful in promoting regimen adherence or in meaning-making processes (e.g., Cattich \& Knudson-Martin, 2009).

\section{DISCUSSION}

In this study, we responded to the call for continued investigation into couples' perspectives of living with diabetes and its challenging selfcare regimen. We developed grounded theory about successful diabetes management that centers on the couple's relationship with diabetes (see Figure 1): their emotions and meanings about diabetes as a life-transforming catalyst for growth, as something to be accepted and accommodated, or as an inconvenience or horror they reject and to which they refuse to attend.

Several couples spoke of their transforming relationship beginning upon a profound scare with diabetes. This is consistent with results reported by Paterson et al. (1999) that transformative experiences seemed most likely to occur upon encountering a significant challenge in diabetes management, "presenting new opportunities for viewing the self and the illness experience" (pp. 794).

Our finding that a partnering comanagement style was most successful is consistent with indications that collaborative coping is beneficial for couples' successful illness management (Berg et al., 2008) and that significant others' beliefs, expressed emotions, and coping strategies predict regimen adherence (Wearden, Tarrier \& Davies, 2000). The current study adds understanding about the specific nature of helpful support: that couples' active partnership of planning and engaging in diabetes management tasks together is most conducive to success.

\section{Limitations/Future Research}

Further research is needed to explore the grounded theory developed here. Qualitative constructs were developed broadly, in order to build a holistic picture of how couples live with the demanding regimen of diabetes. Dimensionality of these constructs should be more deeply investigated; these many interacting constructs are each rich with nuanced complexity.

Also warranting further research is the question of causal direction. This study illustrates that the couple's relationship with diabetes has repercussions in all areas of life with diabetes. It is highly probable that causality is bidirectional, for example, the relationship with diabetes both impacts and is impacted by the degree of success in diabetes management. This paper has 
made a reasonable case for grounded theory about how the studied characteristics interact. A mixed-methods methodology that includes quantitative data and statistical analysis would be useful to analyze causal and hierarchical structure hypotheses.

This paper only begins to explore how couples develop their relationships with diabetes. In our sample, the spouses had a shared relationship with diabetes; possibly, interviewing them together encouraged a single narrative. Further study will be needed to better understand the processes whereby couples negotiate and create their relationships with diabetes, exploring such questions as: how do cultural attitudes, beliefs, and expectations about diabetes shape couples' developing relationships with diabetes? How do issues such as gender and race, which affect the power to define meaning, relate to this process? In what ways is the relationship with diabetes affected by whether it is the husband or the wife who has diabetes?

Participants were geographically situated in Southern California, most indicated their religion was Christian, and the mean age was older than in the general population. In almost a fourth of our sample, at least one spouse worked in a medical/health field, and all of these reported excellent management. This may explain why our sample had higher rates of successful regimen adherence than has been reported elsewhere (Peyrot et al., 2005). Further research is needed to explore how these and other important contextual issues, such as resources and scarcities in financial, educational, social, community, religious, and relational areas, interact with the development and effects of couples' relationships with diabetes.

\section{Clinical Implications}

Given the prevalence of diabetes' and its high comorbidity with psychosocial problems, Marriage and Family Therapists are likely to have many client couples with diabetes over the course of their careers. Being attentive to the particular relationship issues that may arise with these couples can play a pivotal role in the treatment of this population.

In addition, the reported incidence of poor regimen adherence among diabetics is a matter of grave concern. Findings that unsuccessful management is strongly related to psychosocial factors suggest that clinical goals related to diabetes management may arise, particularly for medical family therapists. It may be helpful to consider the centrality of the relationship with diabetes to experiences with management and to work toward coconstructing more accepting or transforming relationships with diabetes. Clinicians may also find that addressing partnering, self-responsibility, interactions with medical professionals, and diabetes-related interactions with family and friends may be beneficial for diabetes management.

Because this study illuminates some of the processes whereby people struggle with diet and exercise, the findings may be helpful to a broader population who deal with obesity and related health issues. We project that it would be beneficial to help clients create more accepting or transforming relationships with health regimens, to encourage couples to actively partner in carrying them out, to ascribe self-responsibility, and to work on improving interactions with medical professionals, family, and friends to accommodate health needs.

This study illustrates how the couple's relationship with diabetes is central to the crucial tasks of diabetes management. We hope these stories of transforming, accepting, and rejecting relationships with diabetes will enhance our understanding about living with this challenging illness. Focus on interactions within the couple's relationship when faced with chronic, life-changing diseases remains a fruitful area of research for Marriage and Family Therapists.

\section{REFERENCES}

Berg, C., Wiebe, D., Butner, J., Bloor, L., Bradstreet, C., Upchurch, R., et al. (2008). Collaborative coping and daily mood in couples dealing with prostate cancer. Psychology and Aging, 23, 505-516.

Campbell, T. L. (2003). The effectiveness of family interventions for physical disorders. Journal of Marital and Family Therapy, 29(2), 263-281.

Cattich, J., \& Knudson-Martin, C. (2009). Spirituality and relationship: A holistic analysis of how couples cope with diabetes. Journal of Marital \& Family Therapy, 35(1), 111-124.

Centers for Disease Control and Prevention. (2009). Diabetes: Successes and opportunities for population-based prevention and control: At a glance 2010. Atlanta, GA: U.S. Department of Health and Human Services, Retrieved from http://www.cdc.gov/nccdphp/publications/aag/pdf/diabetes.pdf. 
Centers for Disease Control and Prevention. (2011). Diabetes: Successes and opportunities for population-based prevention and control: At a glance 2011. Atlanta, GA: U.S. Department of Health and Human Services, CDCP. Retrieved from http://www.cdc.gov/chronicdisease/resources/publications/aag/ddt.htm.

Delamater, A. (2006). Improving patient adherence. Clinical Diabetes, 24, 71.

Denzin, N. K., \& Lincoln, Y. S. (2000). Handbook of qualitative research. 2nd edn. Thousand Oaks, CA: Sage.

Evangelista, L., Kagawa-Singer, M., \& Dracup, K. (2001). Gender differences in health perceptions and meaning in persons living with heart failure. Heart and Lung, 30(3), 167-176.

Fisher, L. (2006). Research on the family and chronic disease among adults: Major trends and directions. Families, Systems \& Health, 24, 373-380.

Ford, M. E., Havstad, S. L., Brooks, B. L., \& Tilley, B. C. (2002). Perceptions of diabetes among patients in an urban health care system. Ethnic Health, 7(4), 243-254.

Gergen, J. (1999). An invitation to social construction. Thousand Oaks: Sage.

Gonder-Frederick, L. A., Cox, D. J., \& Ritterband, L. M. (2002). Diabetes and behavioral medicine: The second decade. Journal of Consulting and Clinical Psychology, 70(3), 611-625.

Hill, M. R., \& Thomas, V. (2000). Strategies for racial identity development: Narratives of black and white women in interracial partner relationships. Family Relations, 2000(49), 193-200.

Hornsten, A., Sandstrom, H., \& Lundman, B. (2004). Personal understandings of illness among people with Type 2 diabetes. Journal of Advanced Nursing, 47(2), 174-182.

Janesick, V. J. (2003). The choreography of qualitative research design: Minuets, improvisations, and crystallization. In N. K. Denzin \& Y. S. Lincoln (Eds), Strategies of qualitative inquiry, 2nd edn pp. 46-79). Thousand Oaks: Sage Publications, Inc.

Johnson, B. D., Dunlap, E., \& Benoit, E. (2010). Structured qualitative research: Organizing "mountains of words" for data analysis, both qualitative and quantitative. Substance Use and Misuse, 45, 648-670.

Kayser, K., Watson, L., \& Andrade, J. (2007). Cancer as a "we-disease": Examining the process of coping from a relational perspective. Families, Systems, \& Health, 25, 404-418.

Kirtland, K. A., Li, Y. F., Geiss, L. S., \& Thompson, T. J. (2008). State-specific incidence of diabetes among adults - participating states, 1995-1997 and 2005-2007. JAMA: Journal of the American Medical Association, 300, 2847-2849.

Kowal, J., Johnson, S. M., \& Lee, A. (2003). Chronic illness in couples: A case for emotionally focused therapy. Journal of Marital and Family Therapy, 29(3), 299-310.

McCaul, K. D., Glasgow, R. E., \& Schafer, L. C. (1987). Diabetes regimen behaviors. Predicting adherence. Medical Care, 25, 868-881.

McDaniel, S., Hepworth, J., \& Doherty, W. (1992). Medical family therapy: A biopsychosocial approach to families with health problems. New York, NY: Basic Books.

Miller, P., Wikoff, R., Keen, O., \& Norton, J. (1987). Health beliefs and regimen adherence of the American Indian diabetic. American Indian and Alaska Native Mental Health Research, 1(1), 24-36.

Murphy, E., \& Kinmonth, A. L. (1995). No symptoms, no problem? Patients' understandings on non-insulin dependent diabetes. Family Practice, 12(2), 184-192.

O’Connor, P. J., Desai, J. R., Solberg, L. I., Rush, W. A., \& Bishop, D. B. (2003). Variation in diabetes care by age: Opportunities for customization of care. BMC Family Practice, 4, 16.

Paterson, B., Thorne, S., Crawford, J., \& Tarko, M. (1999). Living with diabetes as a transformational experience. Qualitative Health Research, 9, 786-802.

Peyrot, M., Rubin, R., Lauritzen, T., Snoek, F., Matthews, D., \& Skovlund, S. (2005). Psychosocial problems and barriers to improved diabetes management: Results of the Cross-National Diabetes Attitudes, Wishes and Needs (DAWN) Study. Diabetic Medicine, 22(10), 1379-1385.

Schwartzberg, S. S. (1993). Struggling for meaning: How HIV-positive gay men make sense of AIDS. Professional Psychology: Research and Practice, 24, 0735-7028.

Skerrett,K.(1998).Coupleadjustmenttotheexperienceofbreastcancer.Families, Systems\&Health,16,281-298.

Snoek, F. J., \& Skinner, T. C. (2002). Psychological counseling in problematic diabetes: Does it help? Diabetic Medicine, 19, 265-273.

Starks, H., \& Trinidad, S. B. (2007). Choose your method: A comparison of phenomenology, discourse analysis, and grounded theory. Qualitative Health Research, 17, 1372-1380.

Strauss, A., \& Corbin, J. (1998). Basics of qualitative research: Techniques and procedures for developing grounded theory. Thousand Oaks, CA: Sage.

Talbot, F., Nouwen, A., Gingras, J., Bélanger, A., \& Audet, J. (1999). Relations of diabetes intrusiveness and personal control to symptoms of depression among adults with diabetes. Health Psychology, 18, 0278-6133.

Trief, P. M., Grant, W., Elbert, K., \& Weinstock, R. (1998). Family environment, glycemic control, and the psychosocial adaptation of adults with diabetes. Diabetes Care, 21, 241-245.

Trief, P. M., Wade, M. J., Britton, K. D., \& Weinstock, R. S. (2002). A prospective analysis of marital relationship factors and quality of life in diabetes. Diabetes Care, 25, 1154-1158. 
Walsh, F. (1998). Strengthening family resilience. New York, NY: The Guilford Press.

Wearden, A., Tarrier, N., \& Davies, R. (2000). Partners' expressed emotion and the control and management of Type 1 diabetes in adults. Journal of Psychosomatic Research, 49(2), 125-130.

Williams, G., Frankel, R., Campbell, T., \& Deci, E. (2000). Research on relationship-centered care and healthcare outcomes from the Rochester Biopsychosocial Program: A self-determination theory integration. Families, Systems \& Health, 18, 79-90.

Williams, G. C., McGregor, H. A., Zeldman, A., Freedman, Z. R., \& Deci, E. L. (2004). Testing a self-determination theory process model for promoting glycemic control through diabetes self-management. Health Psychology, 23, 58-66.

Wynne, L., Shields, C., \& Sirkin, M. (1992). Illness, family theory, and family therapy: I. conceptual issues. amily Process, 31, 3-18. 